\title{
Optimal Vibration Control for Structural Quasi- Hamiltonian Systems with Noised Observations
}

\author{
Zu-guang Ying \\ Department of Mechanics, School of Aeronautics and Astronautics, Zhejiang University, Hangzhou 310027, China
}

Rong-chun Hu

Department of Civil Engineering, College of Civil Engineering and Architecture, Zhejiang University, Hangzhou 310058, China

Rong-hua Huan
Department of Mechanics, School of Aeronautics and Astronautics, Zhejiang University, Hangzhou 310027, China

(Received 19 March 2016; accepted 1 March 2017)

A structural control system with smart sensors and actuators is considered and its basic dynamic equations are given. The controlled, stochastically excited, and dissipative Hamiltonian system with a noised observation is obtained by discretizing the nonlinear stochastic smart structure system. The estimated nonlinear stochastic system with control is obtained, in which the optimally estimated state is determined by the observation based on the extended Kalman filter. Then the dynamical programming equation for the estimated system is obtained based on the stochastic dynamical programming principle. From this, the optimal control law dependent on the estimated state is determined. The proposed optimal control strategy is applied to two nonlinear stochastic systems with controls and noised observations. The control efficacy for stochastic vibration response reductions of the systems is illustrated with numerical results. The proposed optimal control strategy is applicable to general nonlinear stochastic structural systems with smart sensors, smart actuators and noised observations.

\section{INTRODUCTION}

The vibration control for engineering structures subjected to strong random excitations or micro disturbances is a significant research subject. ${ }^{1-3}$ Smart materials have been applied to the structural vibration suppression. For example, magnetorheological materials are used for actuators and piezoelectric materials are used for sensors. ${ }^{4-20}$ A structure system with distributed smart sensors, actuators, and controller is called smart structure, which can sense structural response to external excitations and produce action to control structural response. ${ }^{21-23}$ The dynamics and controls of structures with piezoelectric and magneto-rheological materials have been studied extensively. However, the control performance of the smart structure system depends strongly on the used control strategy. The smart structure system control includes two parts: state estimation based on sensing data and response control based on an estimated state, which are coupled with each other. ${ }^{24-28}$ The stochastic system control with a noised observation is called a partially observable control. Only linear control strategies for partially observable smart structures have been proposed presently.

The smart structure, including multi-degree-of-freedom systems, rods, beams, plates, and shells in modal vibration can be modeled as a controlled, excited, and dissipative Hamiltonian system (or quasi-Hamiltonian system) with observation. ${ }^{29}$ The stochastic optimal controls for linear and nonlinear systems have been studied and many control strategies have been presented. ${ }^{29-46}$ However, the stochastic optimal control for a nonlinear system with a noised observation was only considered in several studies. ${ }^{43}$ Under a specified condition, the separation theorem was applied to convert the nonlinear stochastic system with a noised observation into a completely observable linear system for determining optimal control, but the application is strongly limited. Thus, an approximate estimation and separation strategy is the alternative in practice. The extended Kalman filter is an optimum feasible approximate filter ${ }^{24,28}$ and can be applied to the nonlinear stochastic control system with a noised observation. Based on the extended Kalman filter, the nonlinear stochastic control system with a noised observation is converted into another completely observable nonlinear stochastic system, and then the optimal control law is determined according to the stochastic dynamical programming principle. However, the stochastic optimal control for the partially observable nonlinear stochastic smart structure system (or quasi-Hamiltonian system) has not been studied based on the extended Kalman filter.

In the present paper, the stochastic optimal control for the vibration response reduction of structural quasi-Hamiltonian systems with a noised observations is studied. A new optimal control law expressed by the estimated system state is obtained based on the extended Kalman filter and stochastic dynamical programming principle. Firstly, the differential equations for the structure system with smart sensors and actuators are given. The equations are simplified to ordinary differential equations of the controlled, stochastically-excited, and dissipa- 
tive Hamiltonian system with a noised observation. Secondly, the extended Kalman filter is applied in order to convert the controlled quasi-Hamiltonian system with a noised observation into the nonlinear stochastic control system for the optimally estimated state. Thirdly, the stochastic dynamical programming principle is applied to derive the dynamical programming equation. The optimal control law based on the estimated state is determined by the programming equation. Finally, the proposed optimal control strategy is applied to two nonlinear stochastic systems with controls and noised observations. $\mathrm{Nu}-$ merical results are given to illustrate the control efficacy.

\section{VIBRATION EQUATIONS OF A QUASI-HAMILTONIAN SYSTEM}

Smart materials have been applied recently to the vibration control of engineering structures under strong random excitations. For example, magneto-rheological materials are used for smart actuators whereas piezoelectric materials are used for smart sensors. A structure that has smart devices is a smart system that has the ability to sense structural responses to external excitations and produce actions to control structural response. System state estimations and control strategies are two key problems for effective implementation. In general, the nonlinear dynamic, constitutive, and geometric equations of the main structure can be expressed as ${ }^{47}$

$$
\begin{gathered}
\sigma_{i j, j}+f_{i}-\rho \ddot{u}_{i}-c_{d} \dot{u}_{i}=0 ; \\
\sigma_{i j}=C_{i j k l} \gamma_{k l} ; \\
\gamma_{i j}=\frac{1}{2}\left(u_{i, j}+u_{j, i}+u_{k, i} u_{k, j}\right) ;
\end{gathered}
$$

where $\sigma$ is the stress tensor, $f$ is the body force vector, $u$ is the displacement vector, $\rho$ is the mass density, $c_{d}$ is the damping coefficient, $\gamma$ is the strain tensor, $C$ is the elastic constant tensor, and $i, j, k$, and $l$ denote Cartesian coordinates. The linear dynamic, constitutive, and geometric equations of the sensor structure can be expressed as ${ }^{48}$

$$
\begin{gathered}
\sigma_{i j, j}^{\mathrm{se}}+f_{i}^{\mathrm{se}}-\rho^{\mathrm{se}} \ddot{u}_{i}^{\mathrm{se}}-c_{d}^{\mathrm{se}} \dot{u}_{i}^{\mathrm{se}}=0 \\
\sigma_{i j}^{\mathrm{se}}=C_{i j k l}^{\mathrm{se}} \gamma_{k l}^{\mathrm{se}}+e_{k i j}^{\mathrm{se}} \varphi_{, k}^{\mathrm{se}} ; \\
\gamma_{i j}^{\mathrm{se}}=\frac{1}{2}\left(u_{i, j}^{\mathrm{se}}+u_{j, i}^{\mathrm{se}}\right) ;
\end{gathered}
$$

where the superscript "se" denotes sensor, for example, piezoelectric sensor, $\phi$ is the generalized electric potential and $e$ is the piezoelectric stress constant. The differential equations for the electric displacement vector $D$ are

$$
\begin{gathered}
D_{i, i}^{\mathrm{se}}=\rho_{e m}^{\mathrm{se}} ; \\
D_{i}^{\mathrm{se}}=e_{i k l}^{\mathrm{se}} \gamma_{k l}^{\mathrm{se}}-\varepsilon_{i k}^{\mathrm{se}} \varphi_{, k}^{\mathrm{se}} ;
\end{gathered}
$$

where $\rho_{e m}$ is the free charge density and $\varepsilon$ is the dielectric constant. The mechanical and electrical differential equations for the actuator structure (e.g., piezoelectric actuator) can be expressed as similar to Eqs. (4) to (8), where the superscript se is replaced by ac. Equations (1)-(8) give a basic description of the smart structure system. However, the direct use of the equations for optimal estimation and control is impossible and then their simplification is necessary.
Dominant modes of the main structure with sensors and actuators are used to discretize the structure system in Eqs. (1) to (8), and the Galerkin method is used to eliminate the spatial variables generally. The simplified main structure with actuators is converted into a controlled, excited, and dissipative Hamiltonian system and the sensor structure is converted into an observation system. The inertias of the sensors are small and neglected. The differential equation of the controlled Hamiltonian system with $n$ degree-of-freedoms can be expressed as ${ }^{44}$

$$
\dot{X}=J \frac{\partial H_{a}}{\partial X}+C X+B \Phi_{a}+F W(t)
$$

where $H_{a}$ is the Hamiltonian, $X$ is the state vector, $J$ is the unit symplectic matrix, $C$ is the damping coefficient matrix, $B$ is the control coefficient matrix, $\Phi_{a}$ is the $n_{a}$-dimensional control vector, $F$ is the excitation coefficient matrix, and $W$ is the $m$ dimensional unit intensity excitation vector that is considered to be Gaussian white noise. The equation of the observation system can be expressed as

$$
\Phi_{s}=D X+E W_{s}(t)
$$

where $\Phi_{s}$ is the $n_{s}$-dimensional observation vector, $W_{s}$ is the $m_{s}$-dimensional Gaussian white noise vector with unit intensity, and $D$ and $E$ are coefficient matrices. The observation noise is independent of the white noise excitation.

Equations (9) and (10) describe a nonlinear stochastic quasiHamiltonian control system with a noised observation, which is derived from the smart structure Eqs. (1) to (8). The system state is estimated by using Eq. (10) and the estimated state is used to determine the feedback control for the system in Eq. (9). The optimal estimation and control need to be considered for the nonlinear stochastic system in Eqs. (9) and (10).

\section{STOCHASTIC OPTIMAL ESTIMATION AND CONTROL}

The optimal control of the nonlinear stochastic system (9) and (10) includes the optimal state estimation and the optimal control based on the estimated state, which is called the partially observable optimal control. ${ }^{27}$ The optimal estimation is to find an accurate state by minimizing the estimated error. The error index for the observation in Eq. (10) is given by

$$
J_{F}(\hat{X})=E\left[l_{F}(X-\hat{X}) \mid \Phi_{s}\right]
$$

where $\hat{X}$ is the estimated state, $E[\cdot]$ is the expectation operator, and $l_{F}(\cdot) \geq 0$ is a continuous differentiable function (e.g., a quadratic function). For the nonlinear stochastic system (9) and observation (10) with index (11), the optimally estimated state probability density with infinite dimensions is generally difficult to be obtained exactly, and then an approximate estimation is alternative. The extended Kalman filter is an optimum feasible approximate filter for the nonlinear stochastic system $^{28}$ and is applied to the stochastic optimal control of quasi-Hamiltonian systems with noised observations. Based on the extended Kalman filter, the differential equation for the 
estimated state is obtained from Eq. (9) with observation (10) and index (11) as ${ }^{28}$

$$
\dot{\hat{X}}=J \frac{\partial H_{a}(\hat{X})}{\partial \hat{X}}+C \hat{X}+B \Phi_{a}+R D^{\mathrm{T}} R_{s}^{-1} W_{I}(t)
$$

where $W_{I}$ is the Gaussian white noise vector with covariance matrix $R_{s}=E E^{T}$, and $R$ is the covariance matrix of the estimated error $\tilde{X}=X-\hat{X}$, which is determined by

$$
\begin{aligned}
\dot{R}=\left\{J \frac{\partial^{2} H_{a}(\hat{X})}{\partial \hat{X}^{2}}+\right. & C\} R+R\left\{J \frac{\partial^{2} H_{a}(\hat{X})}{\partial \hat{X}^{2}}+C\right\}^{\mathrm{T}} \\
& +F R_{W} F^{\mathrm{T}}-R D^{\mathrm{T}} R_{s}^{-1} D R ;
\end{aligned}
$$

where $R_{W}=I$ is the identity matrix. Thus, the optimal control of the nonlinear stochastic system in Eqs. (9) and (10) is converted into that of the estimated system in Eq. (12).

The optimal control is to find a control law by minimizing certain performance index. The performance index for the system in Eq. (12) is given by

$$
J_{C}\left(\Phi_{a}\right)=E\left[\int_{0}^{t_{f}} L_{C}\left(\hat{X}(t), \Phi_{a}(t)\right) \mathrm{d} t+\Psi\left(\hat{X}\left(t_{f}\right)\right)\right]
$$

where $L_{C}(\cdot) \geq 0$ is a continuous differentiable function, $\Psi\left(t_{f}\right)$ is a terminal cost and $t_{f}$ is the terminal time. Equations (12) and (14) describe the optimal control problem of the nonlinear stochastic system with the estimated state. Based on the stochastic dynamical programming principle, ${ }^{24,25}$ the dynamical programming equation for system (12) with index (14) is obtained as

$$
\begin{aligned}
& \frac{\partial V}{\partial t}+\min _{\Phi_{a}}\left\{\frac{1}{2} \operatorname{tr}\left(R D^{\mathrm{T}} R_{s}^{-1} D R \frac{\partial^{2} V}{\partial \hat{X}^{2}}\right)\right. \\
& \left.+\left[J \frac{\partial H_{a}}{\partial \hat{X}}+C \hat{X}+B \Phi_{a}\right]^{\mathrm{T}} \frac{\partial V}{\partial \hat{X}}+L_{C}\left(\hat{X}, \Phi_{a}\right)\right\}=0 ;
\end{aligned}
$$

where $V$ is the value function, and $\operatorname{tr}(\cdot)$ is the trace operator. The minimization of the left side of Eq. (15) yields the algebraic equation for the optimal control $\Phi_{a}^{*}$

$$
\frac{\partial L_{C}\left(\hat{X}, \Phi_{a}^{*}\right)}{\partial \Phi_{a}^{*}}+B^{\mathrm{T}} \frac{\partial V}{\partial \hat{X}}=0
$$

The optimal control law is obtained by Eq. (16). For example, for the function with quadratic control $L_{C}=g(\tilde{X})+$ $\Phi_{a}^{\mathrm{T}} R_{C} \Phi_{a}$, where $R_{C}$ is a positive definite symmetric constant matrix and $g(\hat{X}) \geq 0$, it is

$$
\Phi_{a}^{*}=-\frac{1}{2} R_{C}^{-1} B^{\mathrm{T}} \frac{\partial V}{\partial \hat{X}} .
$$

By substituting Eq. (17) into Eq. (15), the value function equation is obtained as

$$
\begin{aligned}
& \frac{\partial V}{\partial t}+\frac{1}{2} \operatorname{tr}\left(R D^{\mathrm{T}} R_{s}^{-1} D R \frac{\partial^{2} V}{\partial \hat{X}^{2}}\right) \\
& +\left[J \frac{\partial H_{a}}{\partial \hat{X}}+C \hat{X}+B \Phi_{a}^{*}\right]^{\mathrm{T}} \frac{\partial V}{\partial \hat{X}}+L_{C}\left(\hat{X}, \Phi_{a}^{*}\right)=0 .
\end{aligned}
$$

Equation (18) can be solved to obtain $V$ and then the optimal control $\Phi_{a}^{*}$ can be determined by Eq. (17), which is based on the estimated state $\hat{X}$. The optimally controlled system is determined by substituting control $\Phi_{a}^{*}$ into Eq. (12), and the controlled state $\hat{X}$ is obtained by solving the equation. The statistics of state $X$ can be calculated by using the state $\hat{X}$ and covariance $R$, which are used for evaluating the control effectiveness.

\section{EXAMPLES AND NUMERICAL RESULTS}

\subsection{Example 1: single-degree-of-freedom nonlinear stochastic control system}

To illustrate the application and effectiveness of the proposed optimal control strategy, we first considered the control for a main mode vibration of geometric nonlinear beams with a piezoelectric sensor and actuator under stochastic excitation. The nonlinear stochastic system with a control and noised observation can be expressed as

$$
\begin{gathered}
\ddot{q}+c \dot{q}+k_{1} q+k_{3} q^{3}=b \varphi_{a}+e_{0} W(t) ; \\
\varphi_{s}=d q+e_{1} W_{s}(t) ;
\end{gathered}
$$

where $q$ is the generalized displacement, $\varphi_{a}$ is the control, $c$, $k_{1}$, and $k_{3}$ are respectively the damping, linear stiffness, and nonlinear stiffness coefficients, $b$ is the control coefficient, $e_{0}$ is the excitation amplitude, $W$ is the Gaussian white noise with unit intensity, $\varphi_{s}$ is the observation, $d$ is the observation coefficient, $e_{1}$ is the observation noise amplitude, and $W_{s}$ is the unit Gaussian white noise. Equations (19) and (20) are rewritten as Eqs. (9) and (10), respectively, where $\Phi_{a}=\varphi_{a}, \Phi_{s}=\varphi_{s}$, $p=\dot{q}$, and

$$
\begin{aligned}
H_{a} & =\frac{1}{2} p^{2}+\frac{1}{2} k_{1} q^{2}+\frac{1}{4} k_{3} q^{4} \\
X & =\left\{\begin{array}{l}
q \\
p
\end{array}\right\} \\
C & =\left[\begin{array}{cc}
0 & 0 \\
0 & -c
\end{array}\right] \\
B & =\left\{\begin{array}{l}
0 \\
b
\end{array}\right\} \\
F & =\left\{\begin{array}{c}
0 \\
e_{0}
\end{array}\right\} \\
D & =\left[\begin{array}{ll}
d & 0
\end{array}\right] \\
E & =e_{1}
\end{aligned}
$$

Applying the extended Kalman filter yields the differential Eq. (12) for the estimated state with the covariance Eq. (13). Equation (12) is converted into the Itô differential equation for the averaged Hamiltonian ${ }^{29}$ by using the stochastic averaging method,

$$
\mathrm{d} \hat{H}_{a}=\left[m_{h}\left(\hat{H}_{a}\right)+<b \varphi_{a} \frac{\partial \hat{H}_{a}}{\partial p}>\right] \mathrm{d} t+\sigma_{h}\left(\hat{H}_{a}\right) \mathrm{d} \xi(t) ;
$$

where $m_{h}$ and $\sigma_{h}$ are the drift and diffusion coefficients, respectively, $\xi$ is the unit Wiener process, and $<\cdot>$ is the averaging operator. According to the stochastic dynamical programming principle, the dynamical programming equation 


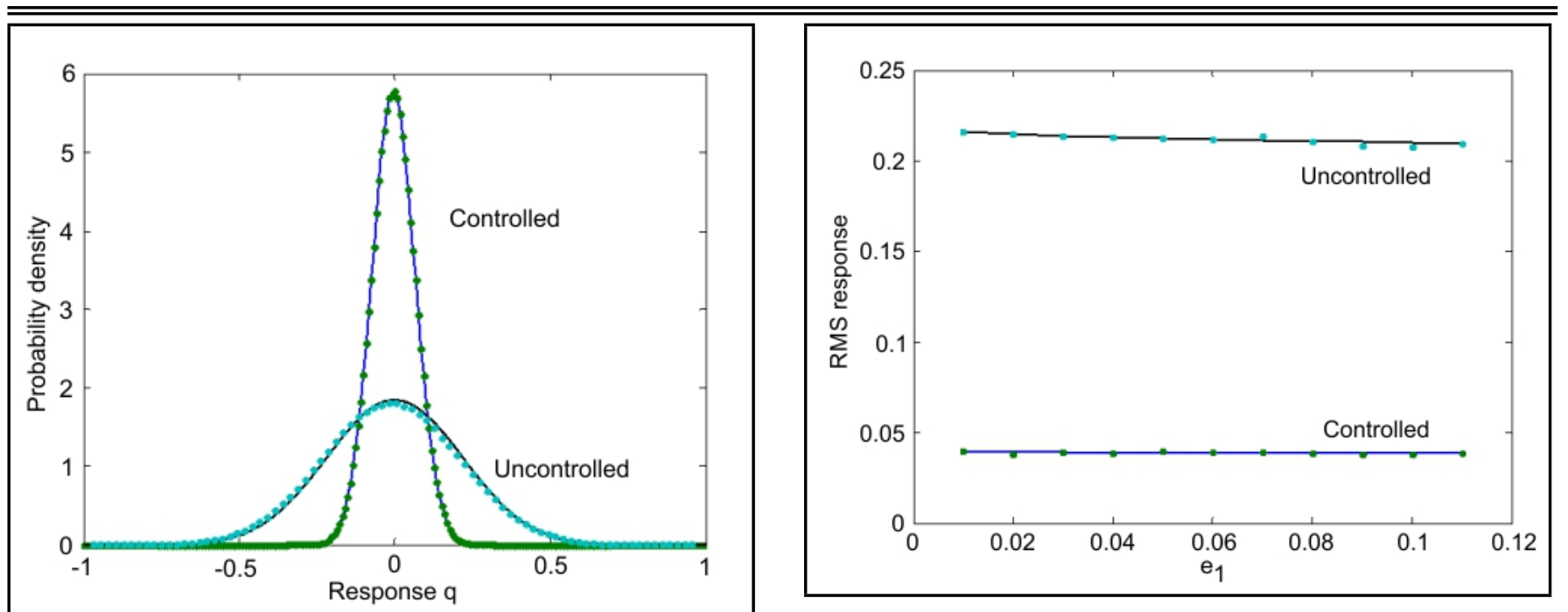

(a)

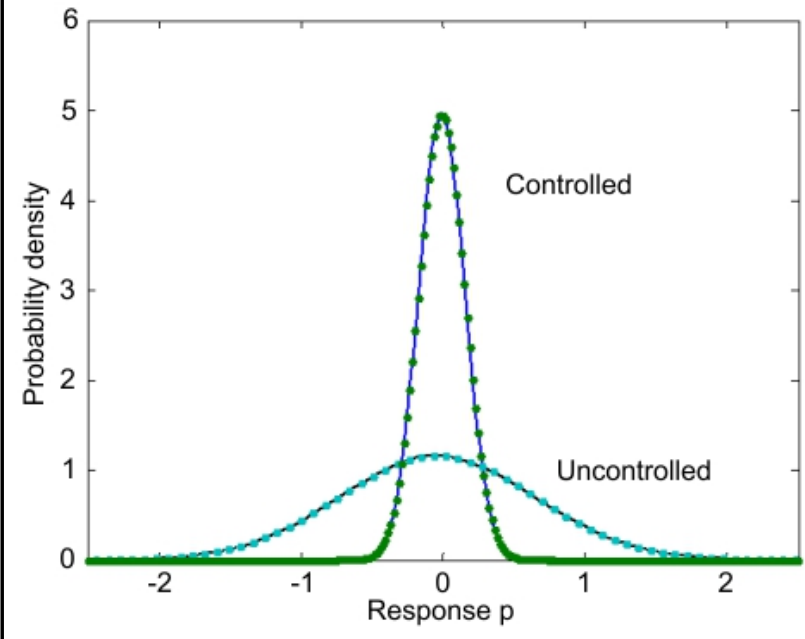

(b)

Figure 1. Controlled and uncontrolled probability densities (solid line: analytical; dot: simulated). The (a) Probability densities of displacement $q$ and (b) Probability densities of velocity $p$.

similar to Eq. (15) is obtained. The optimal control law (17) leads to

$$
\varphi_{a}^{*}=-\frac{b p}{2 R_{C}} \frac{\mathrm{d} V}{\mathrm{~d} \hat{H}_{a}} .
$$

The corresponding stationary value function equation is

$$
\begin{aligned}
\frac{1}{2} \sigma_{h}^{2}\left(\hat{H}_{a}\right) \frac{\mathrm{d}^{2} V}{\mathrm{~d} \hat{H}_{a}^{2}}+m_{h}\left(\hat{H}_{a}\right) \frac{\mathrm{d} V}{\mathrm{~d} \hat{H}_{a}} \\
\quad-\frac{b^{2}}{4 R_{C}}<p^{2}>\left(\frac{\mathrm{d} V}{\mathrm{~d} \hat{H}_{a}}\right)^{2}+g\left(\hat{H}_{a}\right)=\gamma_{0}
\end{aligned}
$$

where $\gamma_{0}$ is a constant. The optimal control is determined finally by Eq. (23) with (24). Then the controlled response and its statistics can be obtained by solving Eq. (12) with Eq. (20) numerically. The analytical system responses can be evaluated by using the probability density, which is obtained by solving the Fokker-Planck-Kolmogorov equation associated with the Itô in Eq. (22). ${ }^{29}$ The proposed optimal control efficacy for the nonlinear stochastic system with a noised observation is evaluated based on the response statistics.

The numerical results on the control system (19) and observation (20) with $c=1.0, k_{1}=10.0, k_{3}=3.0, e_{0}=1.0$,
Figure 2. Controlled and uncontrolled RMS responses $q$ for various observation noise amplitudes $e_{1}$ (solid line: analytical; dot: simulated).

$b=1.0, d=100.0, e_{1}=0.03$ and the quadratic control coefficient $S_{c 2}=1.0$ (i.e., coefficient of linear term of function $g$ ) are obtained and shown in Figs. 1 to 10. The probability densities of the controlled and uncontrolled generalized displacement $q$ and velocity $p$ responses are shown in Figs. 1(a) and 1(b), respectively. The controlled response probability density near zero is larger than the uncontrolled probability density so that the controlled response is reduced. Figure 2 illustrates that the controlled Root-Mean-Square (RMS) displacement response is smaller than the uncontrolled response and the controlled RMS response decreases slightly as the observation noise amplitude $e_{1}$ increases. The corresponding RMS optimal control $\varphi_{a}^{*}$ varying with $e_{1}$ is shown in Fig. 10. Figure 3 illustrates the relative RMS response reduction $K>80 \%$ and the relative reduction per unit RMS control $\mu$. The relative RMS response reduction $K$ is the ratio of absolute difference of controlled and uncontrolled RMS responses to uncontrolled RMS response, and the relative reduction per unit RMS control $\mu$ is the ratio of the relative RMS response reduction to RMS control. The stochastic system response is reduced largely by using the proposed optimal control for various observation noise amplitudes. Figure 4 illustrates that the controlled RMS displacement response is smaller than the uncontrolled response for various observation coefficients $d$. Figure 5 illustrates the large relative RMS response reduction $K$ and the small relative reduction per unit RMS control $\mu$.

Figure 6 illustrates that the controlled RMS displacement response is smaller than the uncontrolled response, and the controlled RMS response has small increment relative to the uncontrolled response with the excitation amplitude $e_{0}$. Figure 7 illustrates the large relative RMS response reduction $K$ and the small relative reduction per unit RMS control $\mu$ for various excitation amplitudes. Figure 8 illustrates that the controlled RMS displacement response decreases as the nonlinear stiffness coefficient $k_{3}$ increases and the controlled RMS response is smaller than the uncontrolled response. The corresponding RMS optimal control $\varphi_{a}^{*}$ varying with $k_{3}$ is shown in Fig. 10. Figure 9 illustrates the large relative RMS response reduction $K$ for various nonlinear stiffness coefficients. Therefore, the proposed optimal control can reduce largely the stochastic vi- 


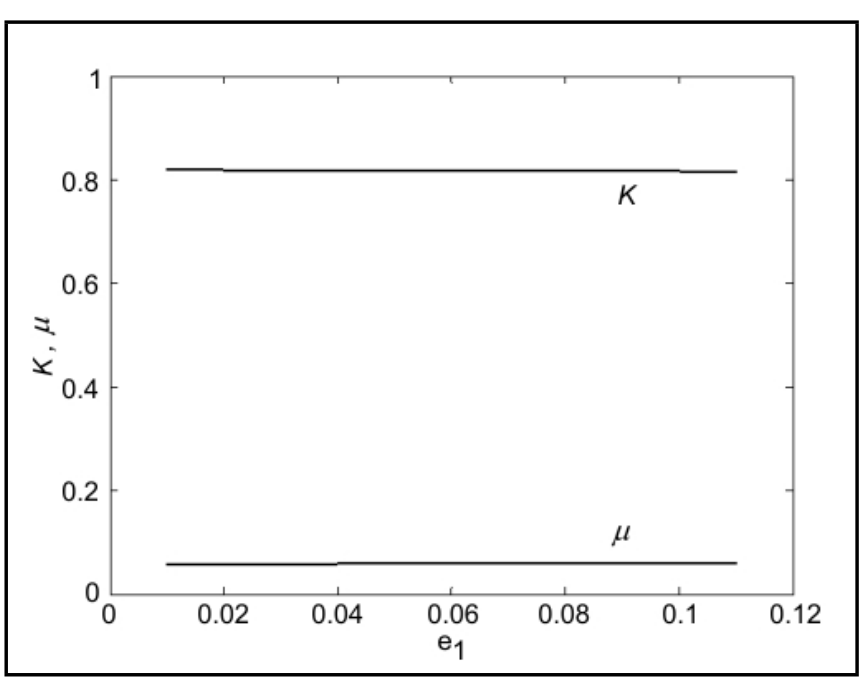

Figure 3. Relative response reduction $K$ and its ratio to control $\mu$ for various observation noise amplitudes $e_{1}$.

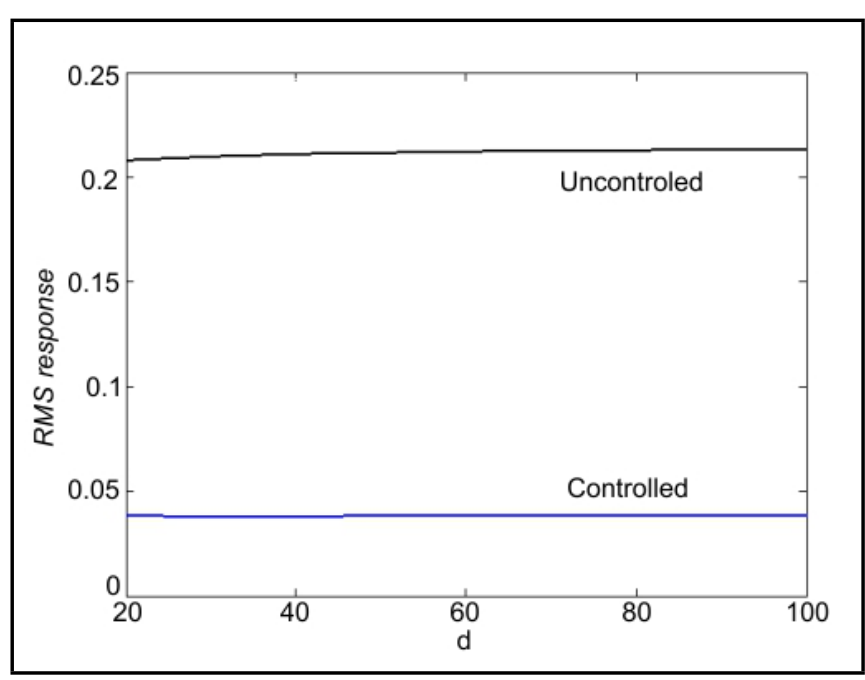

Figure 4. Controlled and uncontrolled RMS responses $q$ for various observation coefficients $d$.

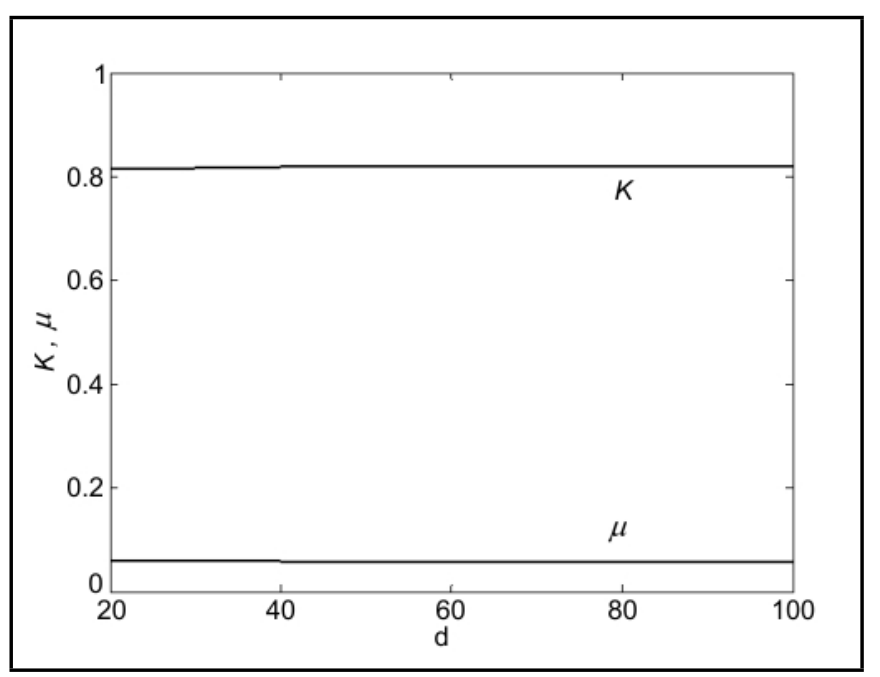

Figure 5. Relative response reduction $K$ and its ratio to control $\mu$ for various observation coefficients $d$.

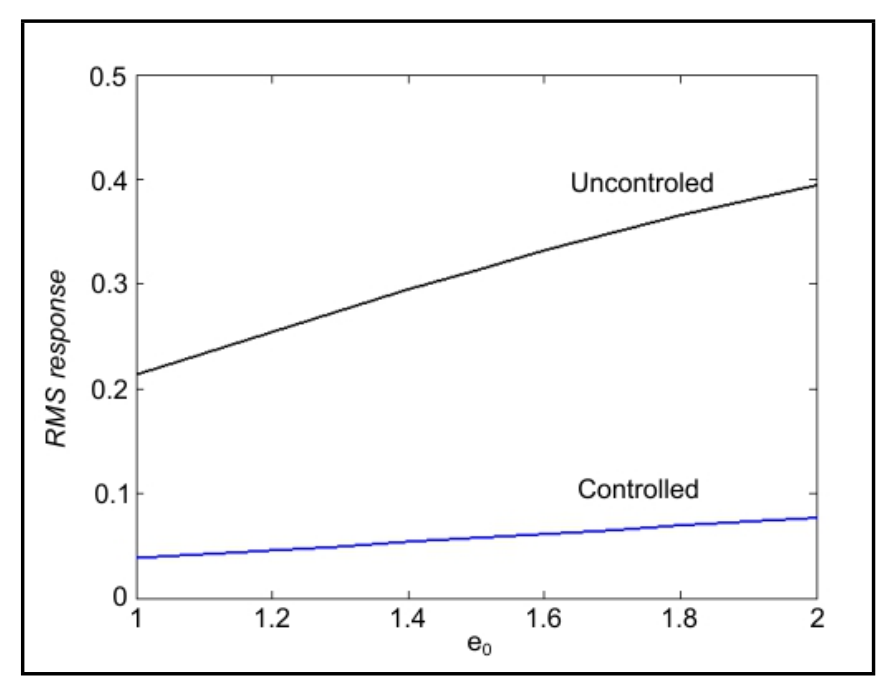

Figure 6. Controlled and uncontrolled RMS responses $q$ for various excitation amplitudes $e_{0}$.

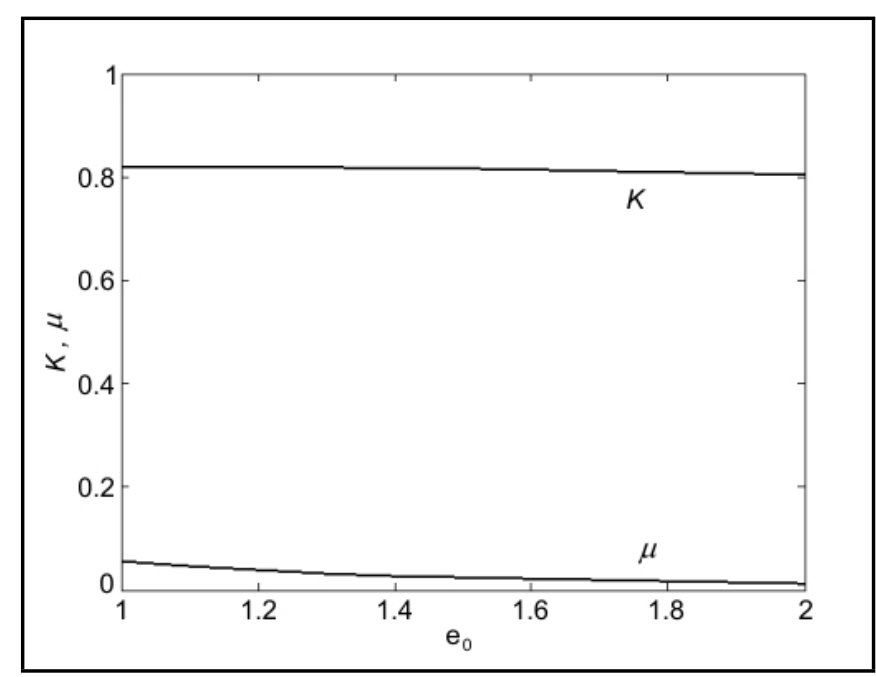

Figure 7. Relative response reduction $K$ and its ratio to control $\mu$ for various excitation amplitudes $e_{0}$.

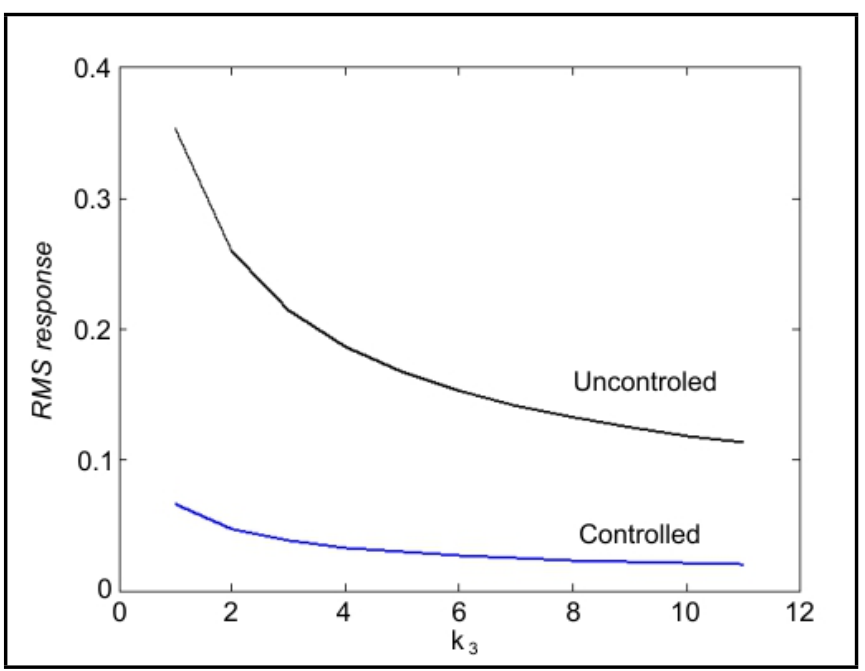

Figure 8. Controlled and uncontrolled RMS responses $q$ for various nonlinear stiffness coefficients $k_{3}$. 


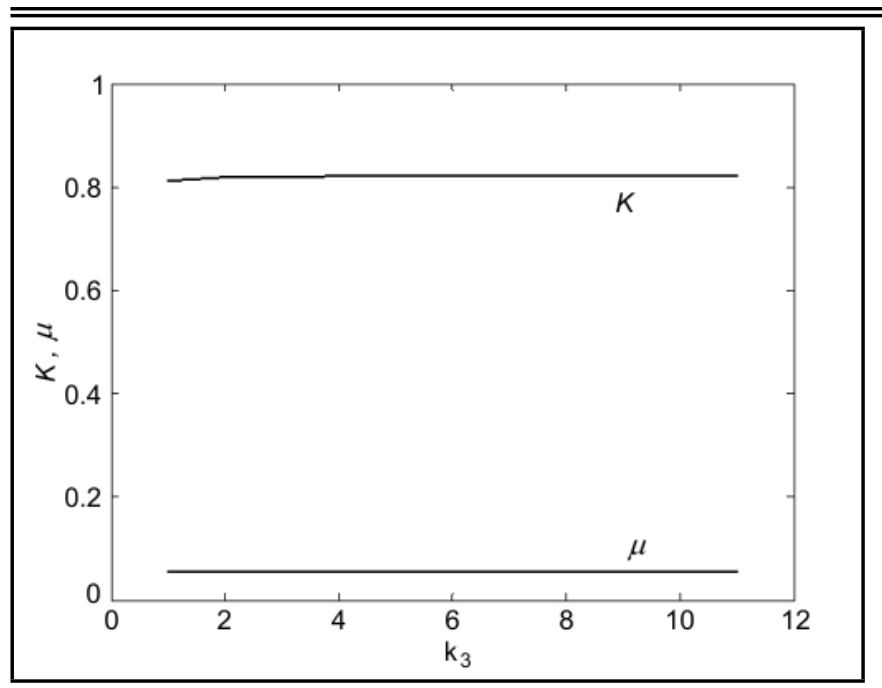

Figure 9. Relative response reduction $K$ and its ratio to control $\mu$ for various nonlinear stiffness coefficients $k_{3}$.

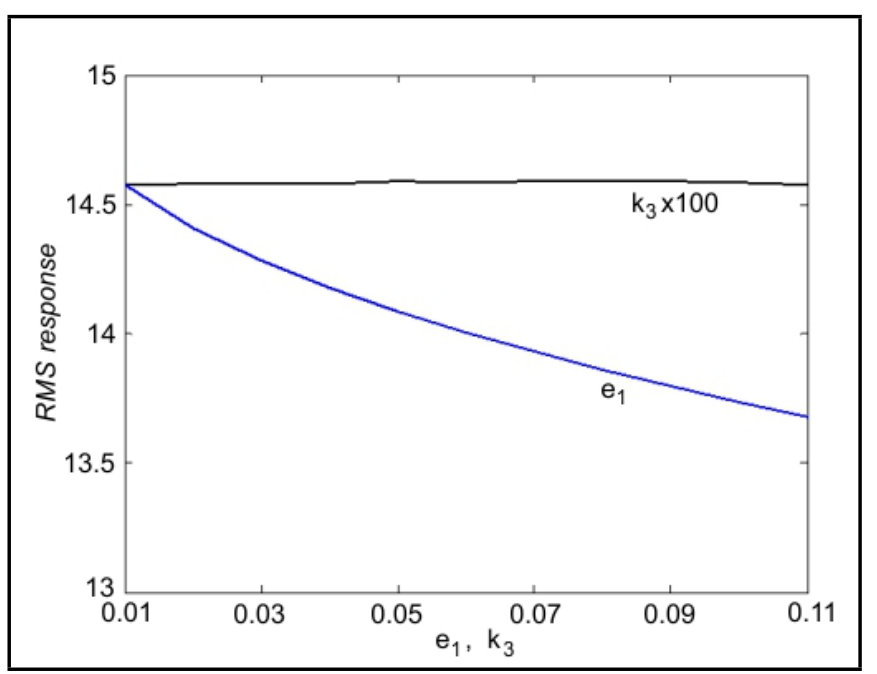

Figure 10. RMS controls $\varphi_{a}^{*}$ for various observation noise amplitudes $e_{1}$ and nonlinear stiffness coefficients $k_{3}$ (note that $k_{3} \times 100$ indicates that the value of $k_{3}$ is the product of horizontal coordinate and 100).

bration response of the nonlinear system (19) with a noised observation (20), and the control effectiveness has good robustness for various system parameters.

\subsection{Example 2: two-degree-of-freedom non- linear stochastic control system}

To further illustrate the application and effectiveness of the proposed optimal control strategy, the control for a two-mainmode coupling vibration of geometric nonlinear beams with piezoelectric sensor and actuator under stochastic excitation was considered. The nonlinear stochastic system with a control and noised observation can be described by Eqs. (9) and (10), in which

$$
\begin{aligned}
& H_{a}=\frac{1}{2}\left(p_{1}^{2}+p_{2}^{2}\right)+\frac{1}{2}\left(k_{11} q_{1}^{2}+k_{12} q_{2}^{2}\right)+k_{2} q_{1} q_{2} \\
& +\frac{1}{4} k_{3}\left(q_{1}-q_{2}\right)^{4} \\
& X=\left[\begin{array}{llll}
q_{1} & q_{2} & p_{1} & p_{2}
\end{array}\right]^{\mathrm{T}}, \\
& \Phi_{a}=\left[\varphi_{a 1}, \varphi_{a 2}\right]^{\mathrm{T}} \text {, }
\end{aligned}
$$

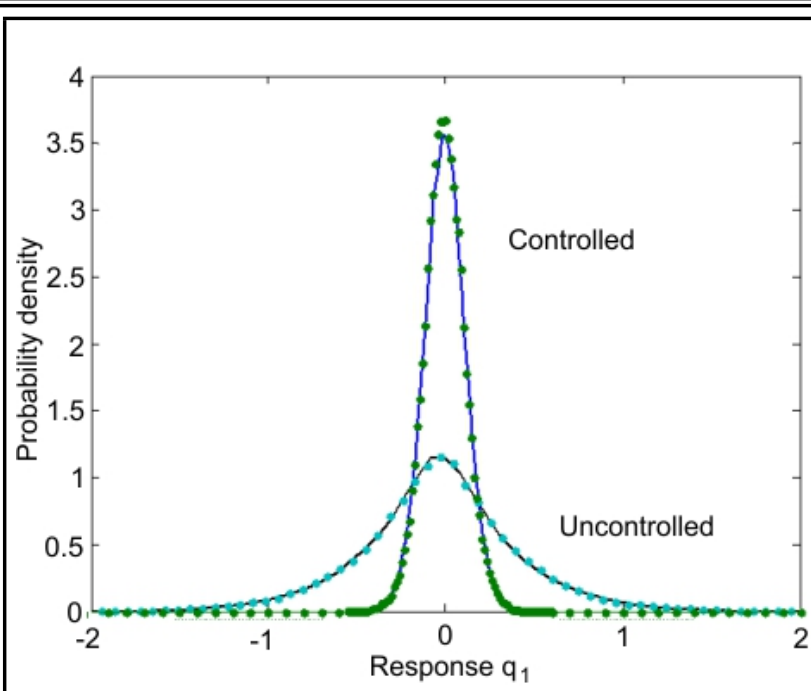

(a)

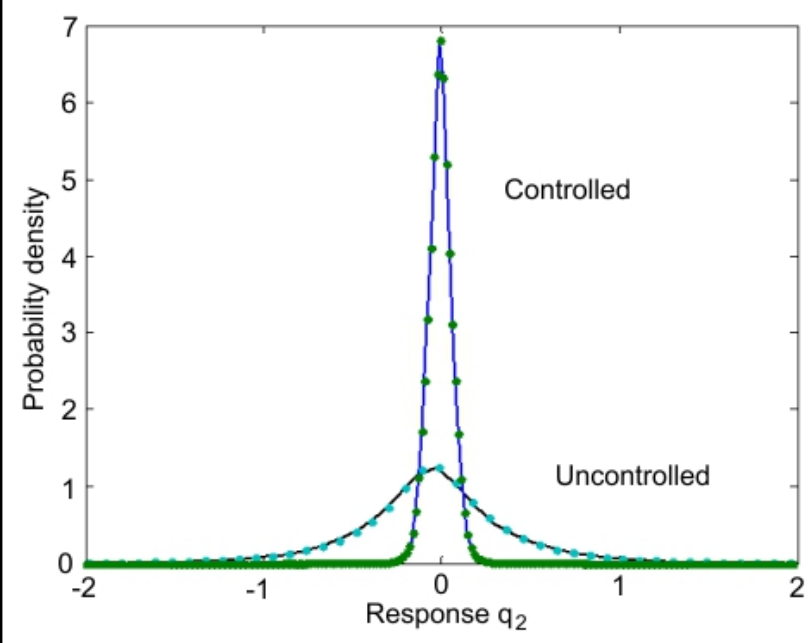

(b)

Figure 11. Controlled and uncontrolled probability densities (solid line: analytical; dot: simulated). The (a) Probability densities of displacement $q_{1}$ and (b) Probability densities of displacement $q_{2}$.

$$
\begin{aligned}
\Phi_{s} & =\left[\varphi_{s 1}, \varphi_{s 2}\right]^{\mathrm{T}}, \\
C & =\operatorname{diag}\left[0,0,-c_{1},-c_{2}\right], \\
F & =\left[\begin{array}{llll}
0 & 0 & e_{01} & e_{02}
\end{array}\right]^{\mathrm{T}}, \\
B & =\left[\begin{array}{llll}
0 & 0 & b_{1} & 0 \\
0 & 0 & 0 & b_{2}
\end{array}\right]^{\mathrm{T}}, \\
D & =\left[\begin{array}{llll}
d_{11} & d_{12} & 0 & 0 \\
d_{12} & d_{22} & 0 & 0
\end{array}\right], \\
E & =\left[\begin{array}{l}
e_{11} \\
e_{12}
\end{array}\right] ;
\end{aligned}
$$

where $q_{i}$ and $p_{i}(i=1,2)$ are respectively the generalized displacement and momentum, $\varphi_{a i}(i=1,2)$ is the control and $\varphi_{s i}(i=1,2)$ is the observation, $c_{i}, k_{1 i}, k_{2}$, and $k_{3}(i=1,2)$ are the damping, linear stiffness, and nonlinear stiffness coefficients, respectively. On the other hand, $b_{i}(i=1,2)$ is the control coefficient, $e_{0 i}(i=1,2)$ is the excitation amplitude, $d_{i j}(i, j=1,2)$ is the observation coefficient, and $e_{1 i}(i=1,2)$ is the observation noise amplitude.

According to the above procedure, using the extended Kalman filter yields the differential Eq. (12) for the estimated 
state. Using the stochastic averaging method converts Eq. (12) into the Itô differential Eq. (22) for the averaged Hamiltonian. Using the stochastic dynamical programming principle yields the dynamical programming equation similar to that in example 1. The optimal control law (17) leads to

$$
\varphi_{a i}^{*}=-\frac{1}{2} R_{C i j}^{-1} b_{j} p_{j} \frac{\mathrm{d} V}{\mathrm{~d} \hat{H}_{a}} .
$$

The corresponding stationary value function equation is

$$
\begin{aligned}
& \frac{1}{2} \sigma_{h}^{2}\left(\hat{H}_{a}\right) \frac{\mathrm{d}^{2} V}{\mathrm{~d} \hat{H}_{a}^{2}}+m_{h}\left(\hat{H}_{a}\right) \frac{\mathrm{d} V}{\mathrm{~d} \hat{H}_{a}} \\
& \quad-\frac{1}{4}<b_{i} p_{i} R_{C i j}^{-1} b_{j} p_{j}>\left(\frac{\mathrm{d} V}{\mathrm{~d} \hat{H}_{a}}\right)^{2}+g\left(\hat{H}_{a}\right)=\gamma_{0} .
\end{aligned}
$$

The optimal control is determined finally by Eq. (26) with (27). Then the controlled response and its statistics can be obtained by solving Eq. (12) with (20) or solving the Fokker-Planck-Kolmogorov equation associated with the It $\hat{o}$ Eq. (22). The proposed optimal control efficacy for the nonlinear stochastic system with a noised observation is evaluated based on the response statistics.

Numerical results on the control system and observation with $c_{1}=1.0, c_{2}=5.0, k_{11}=1.0, k_{12}=4.0, k_{2}=0.01$, $k_{3}=1.0, e_{01}=1.0, e_{02}=0.3, b_{1}=1.0, b_{2}=0.5$, $d_{11}=100.0, d_{12}=10.0, d_{22}=80.0, e_{11}=0.03, e_{12}=0.03$ and the quadratic control coefficient $S_{c 2}=1.0$ (i.e., coefficient of linear term of function $g$ ) are obtained and shown in Figs. 11 to 13 . The probability densities of the controlled and uncontrolled generalized displacement responses $\left(q_{1}\right.$ and $\left.q_{2}\right)$ are shown in Figs. 11(a) and 11(b), respectively. The controlled response probability density near zero is larger than the uncontrolled probability density. Figure 12 illustrates that the relative reduction $K$ of the controlled RMS displacement response $q_{1}$ compared with the uncontrolled response and the relative reduction per unit RMS control $\mu$ vary with the observation noise amplitude $e_{11}$. Figure 13 illustrates that the relative reduction $K$ of the controlled RMS displacement response $q_{1}$ and the relative reduction per unit RMS control $\mu$ vary with the observation coefficients $d_{11}$. It is seen again that the stochastic vibration response of the two-mode coupling nonlinear system with a noised observation is reduced largely by using the proposed optimal control.

\section{CONCLUSIONS}

The basic dynamic equations for a nonlinear stochastic control structure system with smart sensors and actuators have been given and simplified to the controlled, stochasticallyexcited, and dissipative Hamiltonian system with a noised observation. The optimally estimated nonlinear system with control and stochastic excitation has been determined based on the extended Kalman filter. The dynamical programming equation for the estimated system has been obtained based on the stochastic dynamical programming principle, and the optimal control law has been determined by the programming equation. The proposed optimal control strategy has been applied to two nonlinear stochastic systems with controls and noised

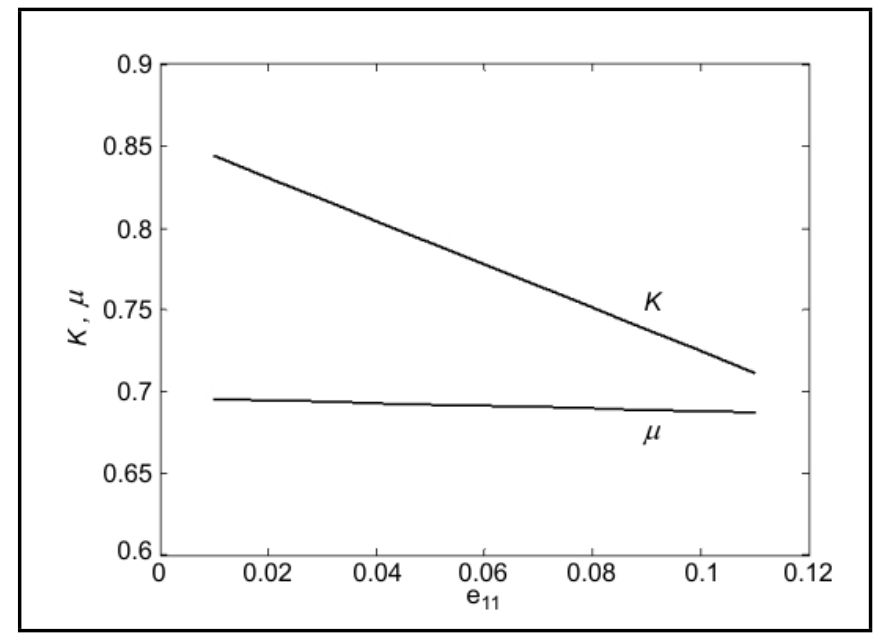

Figure 12. Relative response reduction $K$ and its ratio to control $\mu$ for various observation noise amplitudes $e_{11}$.

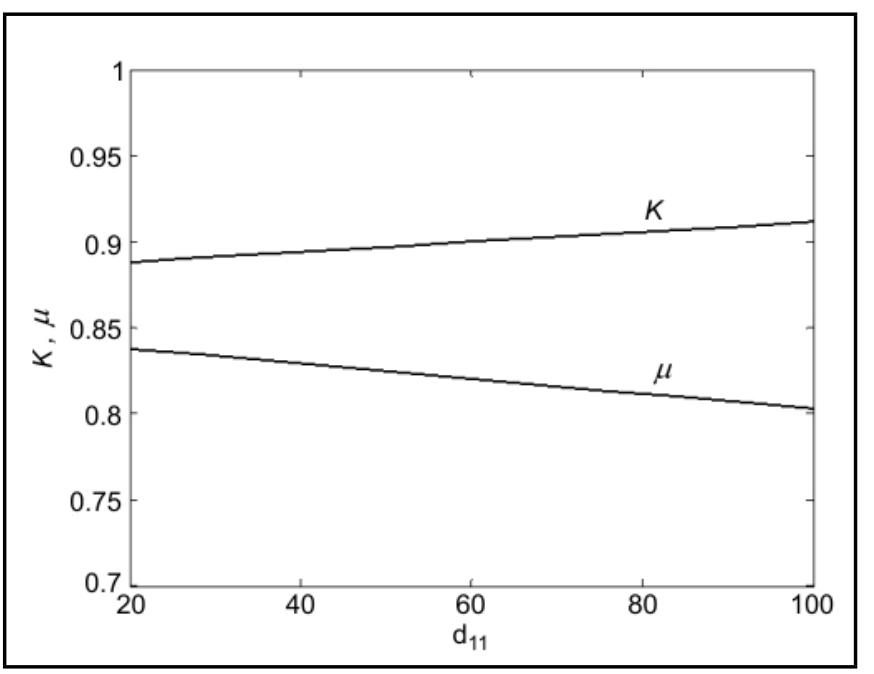

Figure 13. Relative response reduction $K$ and its ratio to control $\mu$ for various observation coefficients $d_{11}$.

observations. Numerical results have illustrated that the proposed optimal control can reduce largely the stochastic vibration response of nonlinear systems with noised observations, and the control effectiveness is insensitive to varying system parameters such as observation and nonlinear coefficients. The proposed optimal control strategy is feasible and effective for the vibration response reduction of nonlinear stochastic smart structure systems with noised observations.

\section{ACKNOWLEDGEMENTS}

This work was supported by the National Natural Science Foundation of China under grant nos. 11572279, 11432012 and 11602216, and the Zhejiang Provincial Natural Science Foundation of China under grant no. LY15A020001.

\section{REFERENCES}

1 Housner, G. W., Bergman, L. A., Caughey, T. K., Chassiakos, A. G., Claus, R. O., Masri, S. F., Skelton, R. E., Soong, T. T., Spencer, B. F., and Yao, J. T. P. Structural control: Past, present, and future, ASCE 
Journal of Engineering Mechanic, 123, 897-971, (1997). https://doi.org/10.1061/(asce)0733-9399(1997)123:9(897)

2 Soong, T. T. and Spencer, B. F. Supplemental energy dissipation: state-of-the-art and state-of-thepractice, Engineering Structures, 24, 243-259, (2002). https://doi.org/10.1016/S0141-0296(01)00092-X

3 Soong, T. T. and Cimellaro, G. P. Future directions in structural control, Structural Control and Health Monitoring, 16, 7-16, (2009). https://doi.org/10.1002/stc.291

4 Casciati, F., Rodellar, J., and Yildirim, U. Active and semi-active control of structures theory and application: A review of recent advances, Journal of Intelligent Material Systems and Structures, 23, 1181-1195, (2012). https://doi.org/10.1177/1045389X12445029

5 Spencer, B. F. and Nagarajaiah, S. State of the art of structural control, ASCE Journal of Structural Engineering, 129, 845-856, (2003). https://doi.org/10.1061/(asce)07339445(2003)129:7(845)

6 Dyke, S. J., Spencer, B. F., Sain, M. K., and Carlson, J. D. Modeling and control of magnetorheological dampers for seismic response reduction, Smart Materials and Structures, 5, 565-575, (1996). https://doi.org/10.1088/0964$1726 / 5 / 5 / 006$

7 York, D., Wang, X. and Gordaninejad, F. A new MR fluid-elastomer vibration isolator, Journal of Intelligent Material Systems and Structures, 18, 1221-1225, (2007). https://doi.org/10.1177/1045389X07083622

8 Zhou, G. Y. and Wang, Q. Study on the adjustable rigidity of magnetorheological-elastomer-based sandwich beams, Smart Materials and Structures, 15, 59-74, (2006). https://doi.org/10.1088/0964-1726/15/1/035

9 Choi, W. J., Xiong, Y. P. and Shenoi, R. A. Vibration characteristics of sandwich beam with steel skins and magnetorheological elastomer cores, Advances in Structural Engineering, 13, 837-847, (2010). https://doi.org/10.1260/13694332.13.5.837

10 Rajamohan, V., Rakheja, S., and Sedaghati, R. Vibration analysis of a partially treated multi-layer beam with magnetorheological fluid, Journal of Sound and Vibration, 329, 3451-3469, (2010). https://doi.org/10.1016/j.jsv.2010.03.010

11 Ying, Z. G., Ni, Y. Q., and Huan, R. H. Stochastic microvibration response analysis of a magnetorheological viscoelastomer based sandwich beam under localized magnetic fields, Applied Mathematical Modelling, 39, 55595566, (2015). https://doi.org/10.1016/j.apm.2015.01.028

12 Nayak, B., Dwivedy, S. K., and Murthy, K. S. R. K. Dynamic analysis of magnetorheological elastomer-based sandwich beam with conductive skins under various boundary conditions, Journal of Sound and Vibration, 330, 18371859, (2011). https://doi.org/10.1016/j.jsv.2010.10.041
13 Ying, Z. G., Ni, Y. Q., and Duan, Y. F. Stochastic micro-vibration response characteristics of a sandwich plate with MR visco-elastomer core and mass, Smart Structures and Systems, 16, 141-162, (2015). https://doi.org/10.12989/sss.2015.16.1.141

14 Rao, S. S. and Sunar, M. Piezoelectricity and its use in disturbance sensing and control of flexible structures: A survey, ASME Applied Mechanics Reviews, 47, 113-123, (1994). https://doi.org/10.1115/1.3111074

15 Narayanan, S. and Balamurugan, V. Finite element modelling of piezolaminated smart structures for active vibration control with distributed sensors and actuators, Journal of Sound and Vibration, 262, 529-562, (2003). https://doi.org/10.1016/S0022-460X(03)00110-X

16 Ray, M. C. Optimal control of laminated shells using piezoelectric sensor and actuator layers, AIAA Journal, 41, 11511157, (2003). https://doi.org/10.2514/2.2058

17 Gupta, V. K., Seshu, P., and Issac, K. K. Finite element and experimental investigation of piezoelectric actuated smart shells, AIAA Journal, 42, 2112-2123, (2004). https://doi.org/10.2514/1.2902

18 To, C. W. S. and Chen, T. Optimal control of random vibration in plate and shell structures with distributed piezoelectric components, International Journal of Mechanical Sciences, 49, 1389-1398, (2007). https://doi.org/10.1016/j.ijmecsci.2007.03.015

19 Jin, Z. L., Yang, Y. W., and Soh, C. K. Semianalytical solutions for optimal distributions of sensors and actuators in smart structure vibration control, Smart Structures and Systems, 6, 767-792, (2010). https://doi.org/10.12989/sss.2010.6.7.767

20 Ying, Z. G., Feng, J., Zhu, W. Q., and Ni, Y. Q. Stochastic optimal control analysis of a piezoelectric shell subjected to stochastic boundary perturbations, Smart Structures and Systems, 9, 231-251, (2012). https://doi.org/10.12989/sss.2012.9.3.231

21 Vepa, R. Dynamics of Smart Structures, John Wiley \& Sons, Chichester, (2010). https://doi.org/10.1002/9780470710623

${ }^{22}$ Fisco, N. R. and Adeli, H. Smart structures: Part I -active and semi-active control, Scientia Iranica A, 18, 275-284, (2011). https://doi.org/10.1016/j.scient.2011.05.034

23 Fisco, N. R. and Adeli, H. Smart structures: Part II -hybrid control systems and control, Scientia Iranica A, 18, 285295, (2011). https://doi.org/10.1016/j.scient.2011.05.035

24 Stengel, R. F. Optimal Control and Estimation, John Wiley \& Sons, New York, (1994).

25 Yong, J. M. and Zhou, X. Y. Stochastic Controls, Hamiltonian Systems and HJB Equations, Springer-Verlag, New York, (1999). https://doi.org/10.1007/978-1-4612-1466-3 
26 Fleming, W. H. and Soner, H. M. Controlled Markov Processes and Viscosity Solutions, Springer, New York, (2006). https://doi.org/10.1007/0-387-31071-1

27 Bensoussan, A. Stochastic Control of Partially Observable Systems, Cambridge University Press, Cambridge, (1992). https://doi.org/10.1017/cbo9780511526503

28 Anderson, B. D. O. and Moore, J. R. Optimal Filtering, Dover Publication, New York, (2005).

29 Zhu, W. Q. Nonlinear stochastic dynamics and control in Hamiltonian formulation, ASME Applied Mechanics Reviews, 59, 230-248, (2006). https://doi.org/10.1115/1.2193137

30 Socha, L. Linearization in analysis of nonlinear stochastic systems: Recent results -part II: application, ASME Applied Mechanics Reviews, 58, 303-315, (2005). https://doi.org/10.1115/1.1995715

31 Datta, T. K. A brief review of stochastic control of structures, Proc. International Symposium on Engineering under Uncertainty, Springer, India, 119-139, (2013). https://doi.org/10.1007/978-81-322-0757-3_6

32 Agrawal, A. K. and Yang, J. N. Optimal polynomial control of seismic-excited linear structures, ASCE Journal of Engineering Mechanics, 122, 753-761, (1996). https://doi.org/10.1061/(asce)0733-9399(1996)122:8(753)

33 Nagarajaiah, S. Adaptive passive, semiactive, smart tuned mass dampers: identification and control using empirical mode decomposition, Hilbert transform, and short-term Fourier transform, Structural Control and Health Monitoring, 16, 800-841, (2009). https://doi.org/10.1002/stc.349

34 Li, J., Peng, Y. B., and Chen, J. B. A physical approach to structural stochastic optimal controls, Probabilistic Engineering Mechanics, 25, 127-141, (2010). https://doi.org/10.1016/j.probengmech.2009.08.006

35 Aldemir, U. Predictive suboptimal semiactive control of earthquake response, Structural Control and Health Monitoring, 17, 654-674, (2010). https://doi.org/10.1002/stc.342

36 Ying, Z. G. and Ni, Y. Q. Optimal control for vibration peak reduction via minimizing large responses, Structural Control and Health Monitoring, 22, 826-846, (2015). https://doi.org/10.1002/stc.1722

37 Socha, L. A. Application of true linearization in stochastic quasi-optimal control problems, Structural Control and Health Monitoring, 7, 219-230, (2000). https://doi.org/10.1002/stc.4300070207

38 Dimentberg, M. F., Iourtchenko, A. S., and Brautus, A. S. Optimal bounded control of steady-state random vibrations, Probabilistic Engineering Mechanics, 15, 381-386, (2000). https://doi.org/10.1016/S0266-8920(00)00008-4
39 Dimentberg, M. F. and Bratus, A. S. Bounded parametric control of random vibrations, Proceedings of Royal Society London A, 456, 2351-2363, (2000). https://doi.org/10.1098/rspa.2000.0615

40 Crespo, L. G. and Sun, J. Q. Stochastic optimal control of nonlinear dynamical systems via Bellmans principle and cell mapping, Automatica, 39, 2109-2114, (2003). https://doi.org/10.1016/S0005-1098(03)00238-3

41 Park, J. H. and Min, K. W. Bounded nonlinear stochastic control based the probability distribution for sdof oscillator, Journal of Sound and Vibration, 281, 141-153, (2005). https://doi.org/10.1016/j.jsv.2004.01.008

42 Scruggs, J. T., Taflanidis, A. A., and Iwan, W. D. Nonlinear stochastic controllers for semiactive and regenerative systems with guaranteed quadratic performance bounds -part 1: State feedback control, Structural Control and Health Monitoring, 14, 1101-1120, (2007). https://doi.org/10.1002/stc.196

43 Ying, Z. G. and Zhu, W. Q. A stochastic optimal control strategy for partially observable nonlinear quasiHamiltonian systems, Journal of Sound and Vibration, 310, 184-196, (2008). https://doi.org/10.1016/j.jsv.2007.07.065

44 Ying, Z. G., Luo, Y. M., Zhu, W. Q., Ni, Y. Q., and Ko, J. M. A semi-analytical direct optimal control solution for strongly excited and dissipative Hamiltonian systems, Communications in Nonlinear Science and Numerical Simulation, 17, 1956-1964, (2012). https://doi.org/10.1016/j.cnsns.2011.09.010

45 Hu, R. C., Ying, Z. G., and Zhu, W. Q. Stochastic minimax optimal control strategy for uncertain quasi-Hamiltonian systems using stochastic maximum principle, Structural and Multidisciplinary Optimization, 49, 69-80, (2014). https://doi.org/10.1007/s00158-013-0958-x

46 Ying, Z. G., Ni, Y. Q., and Duan, Y. F. Parametric optimal bounded feedback control for smart parameter-controllable composite structures, Journal of Sound and Vibration, 339, 38-55, (2015). https://doi.org/10.1016/j.jsv.2014.11.018

47 Mase, G. E. Continuum Mechanics, McGraw-Hill, New York, (1970).

48 Ying, Z. G. and Ni, Y. Q. Dynamic asymmetry of piezoelectric shell structures, Journal of Sound and Vibration, 332, 3706-3723, (2013). https://doi.org/10.1016/j.jsv.2013.03.002 